'Fundação Oswaldo Cruz (Fiocruz), Escola Nacional de Saúde Pública Sergio Arouca (Ensp) - Rio de Janeiro (RJ), Brasil. sssmarins@yahoo.com.br

2 Fundação Oswaldo Cruz (Fiocruz), Escola Nacional de Saúde Pública Sergio Arouca (Ensp) - Rio de Janeiro (RJ), Brasil. cristiani@ensp.fiocruz.br

${ }^{3}$ Fundação Oswaldo Cruz (Fiocruz), Escola Nacional de Saúde Pública Sergio Arouca (Ensp) - Rio de Janeiro (RJ), Brasil. marciafausto@ensp.fiocruz.br

\section{Núcleo de Apoio à Saúde da Família: proposta nacional e implementação em municípios do Rio de Janeiro}

\author{
The Family Health Support Centre: national proposal and \\ implementation in towns of Rio de Janeiro
}

Shirley Soares da Silva Marins do Patrocínio', Cristiani Vieira Machado², Márcia Cristina

Rodrigues Fausto 3

RESUMO O artigo analisa a implantação do Núcleo de Apoio à Saúde da Família (Nasf) como uma estratégia da política de Atenção Básica brasileira, com base nas diretrizes nacionais e a implementação local. O estudo compreendeu dois eixos: caracterização e implantação nacional do Nasf; e realização de dois estudos de caso municipais no estado do Rio de Janeiro. Os resultados mostraram que a expansão nacional do Nasf foi expressiva, porém desigual no País e no estado do Rio. O processo de implementação local variou conforme o contexto, as condições de gestão, as características da Atenção Básica e com as decisões políticas dos atores municipais, com implicações para o funcionamento dos núcleos.

PALAVRAS-CHAVE Atenção Primária à Saúde; Estratégia Saúde da Família; Políticas públicas de saúde; Sistema Único de Saúde.

ABSTRACT This article analyzes the deployment of the Family Health Support Centre as a strategy under the Brazilian primary health care policy, regards the national guidelines and local implementation. The study focuses on two objectives: the description of the Nasf and its national implementation; and the development of two local case studies in Rio de Janeiro State. The results showed that Nasf growth has been expressive, yet unequal, both nationwide and in Rio de Janeiro. The local implementation process varied according to the context, the management conditions, the primary health care characteristics and to political decisions made by local actors.

KEYWORDS Primary Health Care; Family Health Strategy; Public health Policy; Unified Health System. 


\section{Introdução}

Nas últimas décadas, o tema da Atenção Primária à Saúde (APS) ganhou destaque nas agendas políticas e na produção acadêmica, existindo diferentes concepções sobre o seu papel nos sistemas de saúde.

A APS pode ser interpretada de quatro formas distintas, não excludentes: um conjunto de atividades; um nível de atenção; uma estratégia para a organização do sistema e uma filosofia que deve permear todo o sistema de saúde, relacionada ao reconhecimento do direito à saúde (VUORI, 1986).

Starfield (2004) afirma que a APS deve se basear em tecnologia e métodos práticos, representando o primeiro nível de contato dos indivíduos, famílias e comunidade com o sistema de saúde e elemento fundamental para assegurar o processo de atenção continuada à saúde. A autora propôs quatro atributos para balizar a organização da APS: o primeiro contato, a longitudinalidade, a integralidade e a coordenação.

Quanto à organização do trabalho na atenção primária, Gérvas (2004) identifica certa escassez de estudos comparativos sobre as formas de organização, modalidades de pagamento e composição das equipes profissionais. $\mathrm{O}$ autor reconhece o esforço em alguns países europeus (Espanha, Finlândia, Grécia, Portugal e Suécia) de cooperação interdisciplinar na APS, com presença de diversos profissionais nas equipes, tais como: médicos, enfermeiros, dentistas, assistentes sociais.

No Brasil, a partir dos anos 1990, houve uma expansão dos serviços de Atenção Básica em Saúde (ABS). Isso aconteceu por meio da indução federal do Programa Saúde da Família, que preconizou a formação de equipe mínima de profissionais para a Atenção Primária, composta por médico generalista, enfermeiro, auxiliar ou técnico de enfermagem e agentes comunitários de saúde.

Nos anos 2000, a política nacional passou a incentivar a ampliação dos profissionais envolvidos na saúde da família, além da equipe mínima, mediante normas e incentivos financeiros, por meio da conformação de equipes de saúde bucal a partir de 2001 e pela proposição dos Núcleos de Apoio à Saúde da Família (Nasf) em 2008 (BRASIL, 2008).

O Nasf representa uma proposta federal de inserção de outros profissionais na saúde da família com o objetivo de apoiar as equipes mínimas já existentes, expandir o escopo e melhorar a resolutividade das ações. Dessa forma, constituiu-se uma importante inovação da política nacional nos anos 2000 ao propiciar a expansão e diversificação de profissionais na Atenção Básica $(\mathrm{AB})$ e enfatizar a lógica de apoio matricial nesse âmbito, reiterando a saúde da família como estratégia central para a organização da ABS (CASTRO; MACHADO, 2012).

A proposta do Nasf se fundamenta nas concepções de equipe de referência e de apoio matricial que, para Campos e Domitti (2007) são, ao mesmo tempo, arranjos organizacionais e metodologias para a gestão do trabalho em saúde. Nessa perspectiva, a Equipe de Saúde da Família (EqSF) seria a referência do cuidado para o sujeito e o Nasf configuraria a estratégia de apoio matricial a essa equipe (BRASIL, 2009).

Este artigo apresenta os resultados de uma pesquisa que se propôs a analisar a implantação do Nasf como uma estratégia da política de $\mathrm{AB}$ no Brasil, com base nas diretrizes nacionais e os processos de implementação local. O estudo realizado partiu da hipótese que a adoção da proposta do Nasf implica a reconfiguração da organização do trabalho das equipes locais de $\mathrm{AB}$, podendo vir a assumir diferentes formatos, a depender das opções políticas e das características dos serviços nos distintos municípios.

\section{Metodologia}

O estudo compreendeu dois eixos: (1) descrição do contexto nacional, por meio da caracterização da proposta e da implantação nacional 
do Nasf; e (2) análise da implantação do Nasf no estado do Rio de Janeiro, considerando o panorama estadual e o processo de implementação em dois municípios-caso selecionados.

O recorte temporal do estudo corresponde ao período inicial de implantação do Nasf, de 2008 (ano de publicação da regulamentação do Nasf e início de sua implantação no País) a 2011 (ano de realização da pesquisa de campo nos municípios).

Partiu-se da proposição de Walt (1994) que o estudo das políticas de saúde requer a compreensão do processo político e do conteúdo das políticas. Definiram-se as seguintes dimensões de análise: desenho do Nasf (diretrizes, estratégias, inserção na política de AB); composição profissional dos Nasf (nacional, estadual, municipal); panorama da implantação do Nasf (no plano nacional e estadual, considerando a razão de equipes de Estratégia Saúde da Família - ESF/Nasf); processo de implementação e características do Nasf nos municípios selecionados (estratégias para a implementação, lógica de funcionamento, relação com as EqSF e com a rede de atenção).

O primeiro eixo do estudo - contexto nacional - compreendeu inicialmente a caracterização da análise da proposta do Nasf com base em revisão bibliográfica e em análise documental, considerando os enunciados quanto a seus propósitos, desenho e estratégias de operacionalização no âmbito da política de $A B$. A revisão bibliográfica foi realizada nas bases: Biblioteca Virtual em Saúde, Scientific Eletronic Library Online (Scielo) e Portal de Periódicos da Coordenação de Aperfeiçoamento de Pessoal de Nível Superior (Capes). A análise documental compreendeu documentos e portarias do Ministério da Saúde relativos à $\mathrm{AB}$ e ao Nasf identificados na página institucional e no sistema Saúde Legis.

O panorama nacional de implantação dos Nasf de 2008 a 2011 foi mapeado a partir de dados secundários da evolução quantitativa de EqSF e dos Nasf obtidos da Sala de Apoio à Gestão Estratégica do Ministério da Saúde para o cálculo do indicador 'razão entre EqSF e os Nasf' (número de EqSF/número de Nasf) nos âmbitos nacional, regional e estadual. A proposição desse indicador se baseou no parâmetro nacional de recomendação de um Nasf para cada 8 a 15 EqSF. Os dados de composição profissional dos Nasf para o Brasil foram extraídos do Cadastro Nacional dos Estabelecimentos de Saúde (CNES).

O segundo eixo do estudo enfocou a adesão e implantação do Nasf no estado do Rio de Janeiro, considerando o panorama estadual e dois estudos de casos municipais. Inicialmente, analisaram-se os 33 projetos enviados pelos municípios para cadastramento dos Nasf, de onde se obtiveram os dados de composição profissional dos Nasf no Estado. Realizou-se ainda uma entrevista semiestruturada com um dirigente estadual envolvido no processo de implantação dos Nasf.

De maneira análoga ao diagnóstico nacional, o panorama estadual de implantação do Nasf foi mapeado considerando-se o indicador 'razão entre EqSF e os Nasf' no conjunto do Estado, em suas regiões e municípios. A fonte utilizada para aferir o número de Nasf foram os projetos municipais.

A seleção dos municípios do estado do Rio de Janeiro para estudo de casos considerou os critérios: credenciamento do Nasf pelo Ministério da Saúde há mais de um ano, porte populacional, diversidade de inserção regional e indícios da adoção de modelos de funcionamento distintos (segundo os projetos analisados e informações do dirigente estadual entrevistado). Selecionaram-se dois municípios de diferentes regiões do Estado, sendo um de pequeno porte (até $50 \mathrm{mil} \mathrm{ha-}$ bitantes) e outro de médio porte (entre 50 e 100 mil habitantes).

Os estudos de caso foram realizados nos dois municípios (identificados como A e B), considerando o contexto e o processo como foram implementados os Nasf no nível local, estratégias adotadas, composição profissional e funcionamento dos Nasf. Os métodos 
envolveram análise de documentos municipais, visitas aos Nasf e a realização de entrevistas semiestruturadas, sendo duas entrevistas no município A (com um dirigente de $A B$ e um profissional do Nasf) e três entrevistas no município $\mathrm{B}$ (com um dirigente de $\mathrm{AB}$, um coordenador do Nasf e um profissional de um Nasf).

O projeto foi aprovado por Comitê de Ética em Pesquisa da instituição responsável por meio do Parecer No 224/10.

\section{Resultados e discussão}

\section{O Nasf: a proposta e o contexto ini- cial de implantação nacional}

A proposta do Nasf foi formulada pelo Ministério da Saúde, em debates com outros atores nacionais, com o objetivo de ampliar o escopo da $\mathrm{AB}$ e sua resolutividade, reforçando a ESF. O cerne da proposta é a constituição de equipes compostas por profissionais de saúde relevantes para o cuidado na ABS, mas que não constituíam as equipes mínimas de saúde da família, visando a oferecer apoio a essas equipes e fortalecer algumas linhas de cuidado em saúde (BRASIL, 2009).

Os debates sobre a incorporação e expansão de outras categorias profissionais na $\mathrm{AB}$ que culminaram na proposta do Nasf tiveram início em 2003 no âmbito do Ministério da Saúde, se expressando nas reuniões da Comissão Intergestores Tripartite e no Conselho Nacional de Saúde até 2008, quando a proposta foi lançada oficialmente. Os atores que se destacaram nesse debate foram o Ministério da Saúde, o Conselho Nacional de Secretários de Saúde (Conass), o Conselho Nacional de Secretários Municipais de Saúde (Conasems), o Conselho Nacional de Saúde (CNS) e representantes de entidades profissionais em saúde (CASTRO; MACHADO, 2012).

$\mathrm{O}$ argumento central foi a qualificação da $A B$, na perspectiva de ampliação de sua abrangência e escopo e de maior articulação com a rede de serviços. A proposta reforçou a ideia de que as EqSF deveriam permanecer como porta de entrada do sistema, ao enfatizar a importância da atuação do Nasf na lógica do apoio matricial, e não do atendimento ambulatorial especializado (CASTRO; MACHADO, 2012).

O Nasf é constituído por uma equipe de apoio formada por profissionais de diferentes áreas de conhecimento que atuam nos territórios de responsabilidade das EqSF. Entre os seus propósitos e diretrizes, destacam-se a busca da integralidade da atenção e de organização de linha de cuidado continuado em conjunto com a EqSF e em articulação com outros serviços. A presença de outros profissionais de saúde e especialidades médicas visa a aprimorar o cuidado ao ampliar o arcabouço de conhecimentos na $\mathrm{AB}$ a partir da dimensão técnico-pedagógica do apoio matricial. A partir do trabalho da equipe do Nasf, a porta de entrada pode tornar-se mais resolutiva e promover o acesso mais organizado da população aos outros níveis do sistema de saúde. Nesse sentido, o Nasf pode ser um dispositivo que favoreça uma ABS coordenadora do cuidado e a articulação entre $\mathrm{AB}$ e os outros níveis do sistema (BRASIL, 2009).

A proposta do Nasf foi lançada em 2008, posteriormente à publicação da Política Nacional de Atenção Básica (PNAB) em 2006 (BRASIL, 2006), compreendendo duas modalidades: o Nasf 1 composto por, no mínimo, cinco ocupações profissionais de saúde não coincidentes e o Nasf 2, composto, por no mínino, três (BRASIL, 2008). Em 2010, criou-se a modalidade Nasf 3, voltada a prioridades em saúde mental - atenção a usuários de crack, álcool e outras drogas - em municípios com população inferior a 20.000 habitantes (BRASIL, 2010).

Propôs-se ainda o Nasf intermunicipal para viabilizar a implantação de núcleos por meio de consórcios entre municípios que não atingissem escala para implantá-los isoladamente 
(BRASIL, 2008). No País, foram credenciados 136 Nasf intermunicipais entre 2008 e 2011, ano em que essa modalidade foi extinta.

A PNAB divulgada em 2011 incluiu novas diretrizes para o Nasf, como o aumento, de 13 para 19, das categorias profissionais admitidas para compor o núcleo e o reforço do papel simultaneamente matriciador e assistencial das equipes de apoio. Outras inovações foram: 1) vinculação às equipes de $\mathrm{AB}$ de populações específicas - consultório de rua, equipes ribeirinhas e fluviais - e à Academia da Saúde; 2) flexibilização da carga horária com o objetivo de fixação dos profissionais; 3) extinção do Nasf intermunicipal, mantido o financiamento apenas aos já habilitados; 4) supressão do Nasf 3, que tornou-se automaticamente Nasf 2 (BRASIL, 2012).

Em dezembro de 2011, existiam no território nacional 1.589 núcleos, sendo 1.415 Nasf 1 e 174 Nasf 2, cuja composição profissional é apresentada na gráfico 1 .

Gráfico 1. Número de profissionais de Nasf credenciados, por ocupação profissional - Brasil, 2011

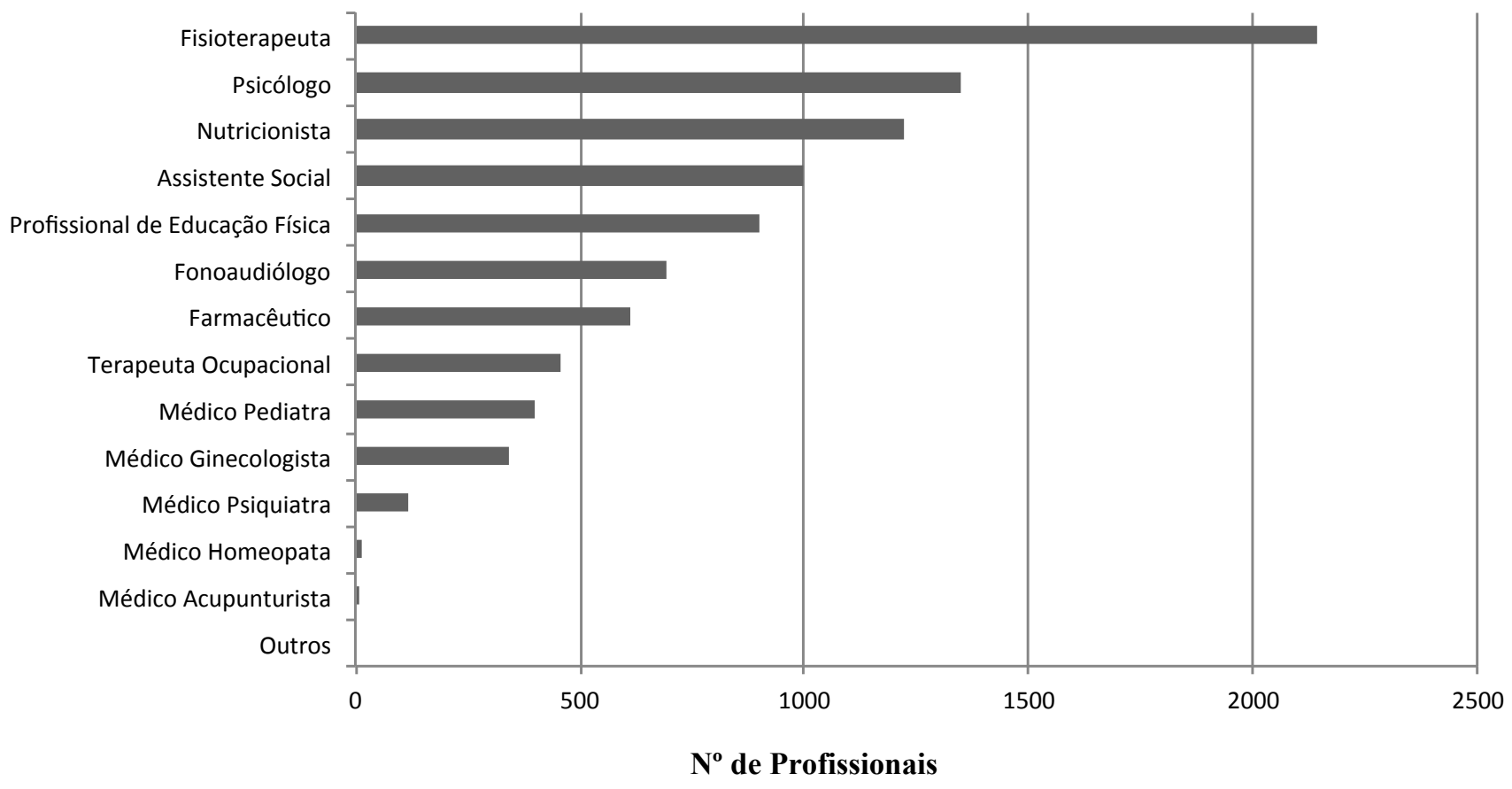

Em relação à composição dos profissionais nos Nasf, observa-se que a categoria profissional mais frequente nacionalmente é o fisioterapeuta, seguida por psicólogo, nutricionista e assistente social. A pequena quantidade das especialidades médicas em relação às outras categorias profissionais da saúde corrobora o resultado de estudo anterior sobre os condicionantes da formulação da proposta, que registrou forte influência da reivindicação de outras categorias para inserção na ESF (CASTRO; MACHADO, 2012). Entretanto, destacam-se dentre os médicos, pediatras, ginecologistas e obstetras, o que pode representar a legitimação desses profissionais, que atuam historicamente no âmbito da ABS.

A adesão ao Nasf foi variada no território nacional, como pode ser visto na tabela 1, que expõe indicadores selecionados de implantação da ESF e do Nasf por Unidades Federadas (UF), regiões e total do Brasil em 2011. 
Tabela 1. Indicadores selecionados de implantação da ESF e do Nasf - UF, regiões, Brasil - 2011

\begin{tabular}{|c|c|c|c|c|c|c|c|c|}
\hline Região & UF & $\begin{array}{l}\text { População } \\
\text { Estimada }\end{array}$ & $\begin{array}{c}\text { № de } \\
\text { Equipes } \\
\text { de Saúde } \\
\text { da Família }\end{array}$ & $\begin{array}{l}\text { Abrangência } \\
\text { Estimada } \\
\text { ESF } \\
\%\end{array}$ & № Nasf 1 & № Nasf 2 & $\begin{array}{c}\text { № Nasf } \\
\text { Total }\end{array}$ & $\begin{array}{l}\text { Razão Equipe } \\
\text { de Saúde da } \\
\text { Família/ Nasf }\end{array}$ \\
\hline \multirow{7}{*}{ Norte } & $A C$ & 746.386 & 138 & 60,0 & 7 & 2 & 9 & 15,3 \\
\hline & AM & 3.538 .387 & 515 & 48,1 & 30 & 1 & 31 & 16,6 \\
\hline & $\mathrm{AP}$ & 684.309 & 128 & 63,0 & 15 & 2 & 17 & 7,5 \\
\hline & PA & 7.688 .593 & 952 & 41,9 & 52 & 5 & 57 & 16,7 \\
\hline & $\mathrm{RO}$ & 1.576 .455 & 277 & 58,0 & 8 & 0 & 8 & 34,6 \\
\hline & $\mathrm{RR}$ & 460.165 & 79 & 54,6 & 4 & 2 & 6 & 13,1 \\
\hline & TO & 1.400 .892 & 408 & 86,9 & 15 & 4 & 19 & 21,4 \\
\hline \multirow[t]{5}{*}{ Total } & & 15.864 .454 & 2.497 & 50,9 & 131 & 16 & 147 & 16,9 \\
\hline & $\mathrm{AL}$ & 3.143 .384 & 755 & 72,3 & 40 & 9 & 49 & 15,4 \\
\hline & BA & 14.097 .534 & 2.748 & 61,9 & 103 & 45 & 148 & 18,5 \\
\hline & CE & 8.530 .155 & 1.834 & 69,3 & 140 & 10 & 150 & 12,2 \\
\hline & MA & 6.645 .761 & 1.783 & 77,9 & 80 & 11 & 91 & 19,5 \\
\hline \multirow[t]{5}{*}{ Nordeste } & PB & 3.791 .315 & 1.242 & 92,9 & 97 & 11 & 108 & 11,5 \\
\hline & $P E$ & 8.864 .906 & 1.870 & 68,2 & 125 & 11 & 136 & 13,7 \\
\hline & $\mathrm{Pl}$ & 3.140 .328 & 1.108 & 96,6 & 53 & 27 & 80 & 13,8 \\
\hline & $\mathrm{RN}$ & 3.198 .657 & 863 & 75,4 & 53 & 27 & 80 & 10,7 \\
\hline & SE & 2.089.819 & 529 & 81,4 & 9 & 0 & 9 & 58,7 \\
\hline \multirow[t]{2}{*}{ Total } & & 53.081 .950 & 12.732 & 72,5 & 700 & 151 & 851 & 18,1 \\
\hline & DF & 2.609 .998 & 110 & 14,7 & 4 & 0 & 4 & 27,5 \\
\hline \multirow{3}{*}{$\begin{array}{l}\text { Centro- } \\
\text { Oeste }\end{array}$} & $\mathrm{GO}$ & 6.080 .716 & 1.159 & 61,7 & 38 & 14 & 52 & 22,2 \\
\hline & MS & 2.477 .542 & 450 & 60,0 & 16 & 18 & 34 & 13,2 \\
\hline & MT & 3.075 .936 & 589 & 63,2 & 8 & 12 & 20 & 29,4 \\
\hline \multirow[t]{2}{*}{ Total } & & 14.058 .094 & 2.308 & 53,2 & 66 & 44 & 110 & 20,9 \\
\hline & ES & 3.547 .055 & 567 & 51,6 & 4 & 3 & 7 & 81,0 \\
\hline \multirow{3}{*}{ Sudeste } & $M G$ & 19.728 .701 & 4.333 & 69,8 & 227 & 30 & 257 & 16,8 \\
\hline & RJ & 16.112 .678 & 1.880 & 39,4 & 97 & 1 & 98 & 19,1 \\
\hline & SP & 41.587 .182 & 3.524 & 28,7 & 125 & 0 & 125 & 28,1 \\
\hline \multirow[t]{2}{*}{ Total } & & 80.364 .410 & 10.304 & 41,8 & 453 & 34 & 487 & 21,1 \\
\hline & PR & 10.512 .349 & 1.822 & 56,0 & 62 & 6 & 68 & 26,7 \\
\hline \multirow[t]{2}{*}{ Sul } & RS & 10.733 .030 & 1.232 & 36,5 & 16 & 7 & 23 & 53,5 \\
\hline & SC & 6.317 .054 & 1.400 & 70,4 & 42 & 0 & 42 & 33,3 \\
\hline Total & & 27.386 .891 & 4.454 & 51,7 & 120 & 13 & 133 & 33,4 \\
\hline BRASIL & & 190.755.799 & 32.295 & 53,4 & 1415 & 174 & 1.589 & 20,3 \\
\hline
\end{tabular}

Nota: Dados populacionais - IBGE (Acesso em: 20 maio 2012). Demais dados - Sala de Apoio à Gestão Estratégica do Ministério da Saúde (Acesso em: 20 maio 2012). 
Observa-se que, no Brasil, a razão EqSF/ Nasf está acima da faixa recomendada pelo Ministério da Saúde. No País, essa razão é 20,3 quando deveria estar entre 8 e 15, devido à quantidade de EqSF que devem ser vinculadas a cada Nasf. Portanto, os Nasf têm a possibilidade de se expandir no País, com vistas a um adequado atendimento quantitativo às equipes já existentes. Visto que a abrangência populacional da ESF também tem possibilidade de expansão nacional, é possível que o número de Nasf aumente à medida que novas EqSF vierem a ser implantadas.

Em relação às regiões do Brasil, a que apresenta menor razão EqSF/Nasf é a região Norte $(16,9)$, e a que tem o maior razão é a região Sul $(33,4)$. Dessa forma, é possível sugerir que, na primeira, os municípios em média aderiram mais ao Nasf para apoio às EqSF já existentes, enquanto na segunda ainda há elevada possibilidade de aumento do número de Nasf. A maior adesão de credenciamentos no Norte pode estar relacionada às dificuldades anteriores de inserção de outros profissionais de saúde e especialidades médicas básicas, atenuadas pela introdução do incentivo financeiro do Nasf. No entanto, na região Sul, outras formas de organizar a ABS, com a inserção de outros profissionais, já poderiam estar bem desenvolvidas nos municípios, o que pode explicar em parte a menor adesão à proposta no período estudado. Cabe considerar ainda que nenhuma região do País está, em média, dentro da faixa de razão EqSF/ Nasf recomendada pelo Ministério da Saúde.

Quanto às Unidades da Federação, destacam-se o Amapá, por apresentar a menor razão entre número de EqSF e Nasf do País $(7,5)$, e o Espírito Santo, por ter a razão mais elevada de, em média, 81 EqSF para cada Nasf. Somente sete UF estão de acordo com a faixa recomendada para a relação EqSF/Nasf: um estado da região Norte (RR); cinco estados da região Nordeste (CE, PB, PE, PI, RN) e um estado da região Centro-Oeste (MS).

\section{O Nasf no Rio de Janeiro: panorama estadual e implementação local}

O estado do Rio de Janeiro passou por um intenso processo de descentralização em saúde nos anos 1990, quando os municípios assumiram muita responsabilidade na gestão do sistema (VIANNA; LIMA, 2013), principalmente na $\mathrm{AB}$, afirmando a agenda mais geral da política de saúde no País. As características da $\mathrm{AB}$ em saúde no Estado são influenciadas pelas peculiaridades dos municípios, que apresentam diferentes perfis e capacidades de gestão do Sistema Único de Saúde (SUS), o que permite variações na sua organização local.

A adesão dos municípios fluminenses à proposta dos Nasf ocorreu desde o ano de publicação da primeira regulamentação federal. Entre 2008 e 2011, dos 92 municípios do Estado, 34 enviaram projetos de implantação de Nasf para aprovação da Comissão Intergestores Bipartite (CIB)/RJ. Destes, 33 projetos foram aprovados, sendo trinta Nasf 1, um Nasf 2 e dois Nasf 3. Os municípios que aderiram à proposta localizam-se, predominantemente, nas regiões do centro ao sul do estado do Rio de Janeiro, com menor adesão nas regiões serrana e norte fluminense.

A atuação da esfera estadual na implementação dos Nasf foi relativamente restrita, voltada para esclarecimentos e apoio aos municípios para elaboração dos projetos, recebimento e análise das propostas e organização do credenciamento junto à CIB. No entanto, destacou-se a proeminência da equipe estadual de saúde mental, que se articulou com os municípios para incentivar a inserção de profissionais dessa área nos Nasf, o que de fato se refletiu na composição profissional dos núcleos.

A gráfico 2 mostra a distribuição dos profissionais que compunham os Nasf no estado do Rio de Janeiro por ocupação profissional, mapeada a partir dos projetos de Nasf enviados à Secretaria de Estado de Saúde (SES)/RJ. 
Gráfico 2. Número de profissionais do Nasf, pro ocupação - estado do Rio de Janeiro - 2011

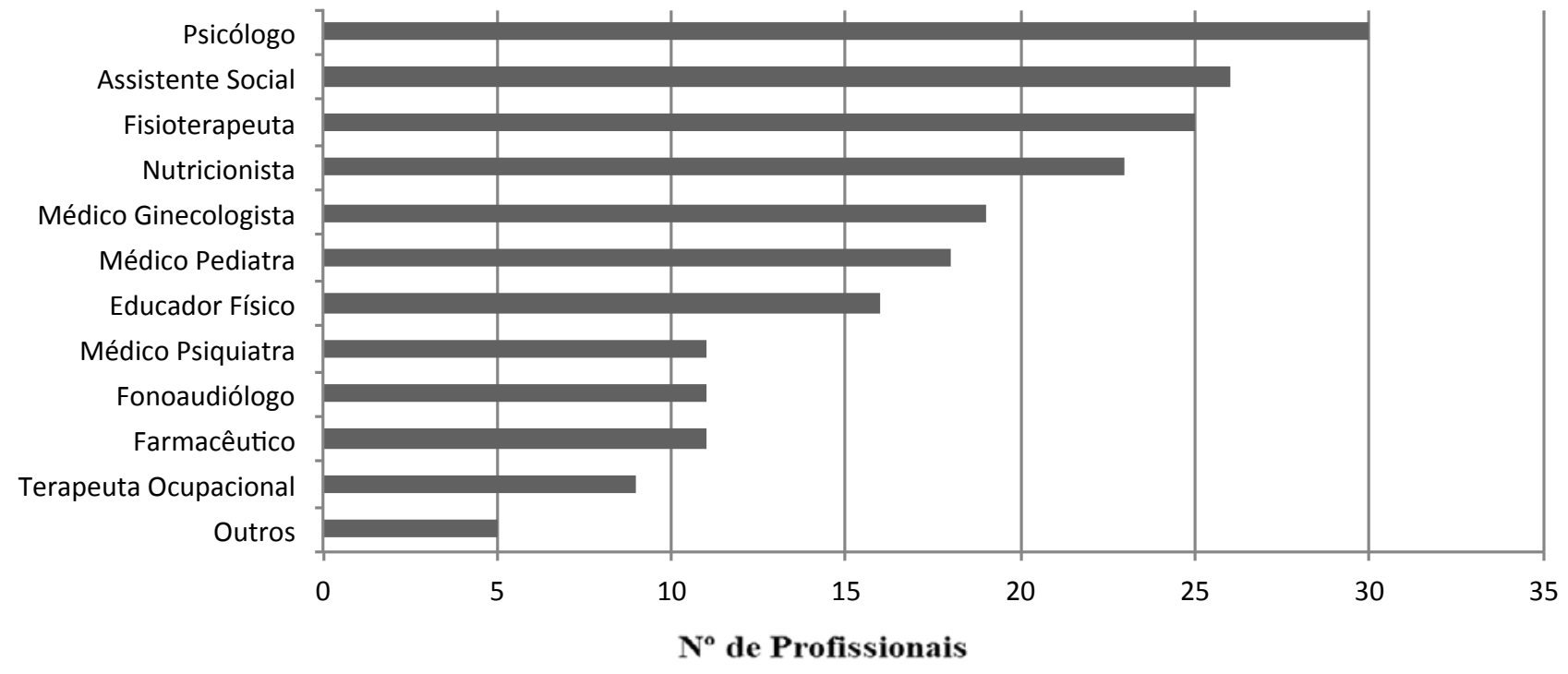

Observou-se que as categorias profissionais mais frequentemente escolhidas no Estado para os Nasf foram: psicólogo, assistente social, fisioterapeuta e nutricionista. Logo em seguida, estavam as especialidades médicas básicas, representadas pelos médicos ginecologista e pediatra. Comparando-se com o perfil nacional de composição profissional, chama a atenção que as quatro profissões mais frequentes tenham sido as mesmas, apenas invertendo-se a ordem de predominância, já que no conjunto Brasil a categoria mais presente no Nasf foi a de fisioterapeuta, seguida de psicólogo, nutricionista e assistente social. Talvez o peso da atuação da equipe de saúde mental no processo de implantação dos Nasf no Estado tenha influenciado a maior frequência de psicólogos no Rio de Janeiro.

A tabela 2 expõe alguns indicadores selecionados de implantação da ESF e do Nasf nos municípios do estado do Rio de Janeiro.

Tabela 2. Indicadores selecionados de implantação da ESF e do Nasf nos municípios do estado do Rio de Janeiro com Nasf implantados - 2011

\begin{tabular}{|c|c|c|c|c|c|c|c|c|}
\hline Região de Saúde & Município & $\begin{array}{l}\text { População } \\
\text { Estimada }\end{array}$ & $\begin{array}{l}\mathrm{N}^{\circ} \text { de } \\
\text { Equipes de } \\
\text { Saúde da } \\
\text { Família }\end{array}$ & $\begin{array}{c}\text { Abrangência } \\
\text { Estimada } \\
\text { ESF } \\
\%\end{array}$ & $\begin{array}{c}\mathrm{N}^{\circ} \\
\text { Nasf } 1\end{array}$ & $\begin{array}{c}\mathrm{N}^{\circ} \\
\text { Nasf } 2\end{array}$ & $\begin{array}{c}\mathrm{N}^{\circ} \text { Nasf } \\
\text { Total }\end{array}$ & $\begin{array}{l}\text { Razão } \\
\text { Equipe de } \\
\text { Saúde da } \\
\text { Família/ } \\
\text { Nasf }\end{array}$ \\
\hline \multirow{2}{*}{ Baía de Ilha Grande } & Angra dos Reis & 173.370 & 37 & 75,3 & 4 & 0 & 4 & 9,2 \\
\hline & Mangaratiba & 37.343 & 11 & 100,0 & 1 & 0 & 1 & 11,0 \\
\hline \multirow{4}{*}{$\begin{array}{l}\text { Baixada } \\
\text { Litorânea }\end{array}$} & Armação dos Búzios & 28.279 & 8 & 100,0 & 1 & 0 & 1 & 8,0 \\
\hline & Casimiro de Abreu & 36.360 & 10 & 97,6 & 1 & 0 & 1 & 10,0 \\
\hline & São Pedro da Aldeia & 89.739 & 12 & 47,1 & 1 & 0 & 1 & 12,0 \\
\hline & Saquarema & 75.906 & 11 & 51,1 & 1 & 0 & 1 & 11,0 \\
\hline Capital & Rio de Janeiro & 6.355 .949 & 506 & 27,6 & 47 & 0 & 47 & 10,7 \\
\hline \multirow{5}{*}{$\begin{array}{l}\text { Centro Sul- } \\
\text { Fluminense }\end{array}$} & Areal & 11.540 & 5 & 100,0 & 1 & 0 & 1 & 5,0 \\
\hline & Eng. Paulo de Frontin & 13.324 & 6 & 100,0 & 1 & 0 & 1 & 6,0 \\
\hline & Mendes & 17.981 & 7 & 100,0 & 1 & 0 & 1 & 7,0 \\
\hline & Paraíba do Sul & 41.367 & 17 & 100,0 & 2 & 0 & 2 & 8,5 \\
\hline & Três Rios & 77.851 & 22 & 94,8 & 2 & 0 & 2 & 11,0 \\
\hline
\end{tabular}


Tabela 2. (cont.)

\begin{tabular}{|c|c|c|c|c|c|c|c|c|}
\hline \multirow{6}{*}{ Médio Paraíba } & Barra Mansa & 178.355 & 31 & 60,1 & 3 & 0 & 3 & 10,3 \\
\hline & Pinheiral & 22.968 & 8 & 100,0 & 1 & 0 & 1 & 8,0 \\
\hline & Piraí & 26.637 & 10 & 100,0 & 1 & 0 & 1 & 10,0 \\
\hline & Rio Claro & 17.517 & 8 & 100,0 & 1 & 0 & 1 & 8,0 \\
\hline & Rio das Flores & 8.633 & 4 & 100,0 & 0 & 1 & 1 & 4,0 \\
\hline & Volta Redonda & 259.012 & 60 & 80,2 & 8 & 0 & 8 & 7,5 \\
\hline $\begin{array}{l}\text { Metropolitana } \\
\text { I }\end{array}$ & Belford Roxo & 472.008 & 32 & 23,5 & 3 & 0 & 3 & 10,6 \\
\hline \multirow{5}{*}{$\begin{array}{l}\text { Metropolitana } \\
\text { II }\end{array}$} & Mesquita & 168.966 & 14 & 28,6 & 1 & 0 & 1 & 14,0 \\
\hline & Seropédica & 79.179 & 16 & 70,6 & 1 & 0 & 1 & 16,0 \\
\hline & Maricá & 131.355 & 14 & 37,8 & 1 & 0 & 1 & 14,0 \\
\hline & São Gonçalo & 1.008 .065 & 166 & 57,2 & 15 & 0 & 15 & 11,0 \\
\hline & Silva Jardim & 21.356 & 8 & 100,0 & 1 & 0 & 1 & 8,0 \\
\hline \multirow{2}{*}{$\begin{array}{l}\text { Norte } \\
\text { Fluminense }\end{array}$} & Macaé & 212.433 & 25 & 41,7 & 3 & 0 & 3 & 8,3 \\
\hline & Quissamã & 20.747 & 8 & 100,0 & 1 & 0 & 1 & 8,0 \\
\hline \multirow{3}{*}{$\begin{array}{l}\text { Noroeste } \\
\text { Fluminense }\end{array}$} & $\begin{array}{l}\text { Bom Jesus do } \\
\text { Itabapoana }\end{array}$ & 35.546 & 10 & 97,4 & 1 & 0 & 1 & 10,0 \\
\hline & Itaperuna & 96.542 & 18 & 64,7 & 2 & 0 & 2 & 9,0 \\
\hline & $\begin{array}{l}\text { Santo Antonio de } \\
\text { Pádua }\end{array}$ & 40.735 & 10 & 85,0 & 1 & 0 & 1 & 10,0 \\
\hline \multirow{2}{*}{ Serrana } & Bom Jardim & 25.539 & 8 & 100,0 & 1 & 0 & 1 & 8,0 \\
\hline & Cachoeiras de Macacu & 54.713 & 9 & 57,2 & 1 & 0 & 1 & 9,0 \\
\hline
\end{tabular}

Nota: Dados populacionais - IBGE. Dados de abrangência populacional estimada das ESF - Sala de Apoio à Gestão Estratégica do Ministério da Saúde (Acesso em: 19 jul. 2012). Dados dos Nasf - Projetos Municipais dos Nasf/SES/RJ.

Observa-se que todos os municípios do estado do Rio de Janeiro que aderiram ao Nasf e que possuem $100 \%$ de abrangência populacional da ESF encontram-se dentro da faixa recomendada pelo governo federal de um Nasf para cada 8 a 15 EqSF. Assim, a possibilidade de expansão adicional dos núcleos nesses municípios é limitada.

Entretanto, os municípios com pequena e média abrangência da ESF têm a possibilidade de ampliá-la, o que também pode significar um aumento das equipes de Nasf. Destaca-se o município de Seropédica, como a maior possibilidade de expansão da proposta Nasf, visto que apresenta relação entre o número de EqSF e Nasf superior ao parâmetro nacional, e possibilidade de aumentar a abrangência da ESF.
Para análise da implantação local do Nasf, realizaram-se dois estudos de caso em municípios fluminenses com características bem diferenciadas. O município A é de pequeno porte, atividade econômica baseada na pesca e turismo, emancipação política e organização do sistema de saúde municipal posterior à Constituição de 1988. Já o município B, apresenta médio porte populacional, atividade econômica centrada no comércio e indústria, é mais antigo, possui sistema de saúde historicamente marcado por características do modelo médico-assistencial anterior à constituição do SUS e presença mais expressiva do setor privado.

O quadro 1 resume as principais características e os resultados da análise de implementação dos Nasf nos dois municípios do estudo de casos. 
Quadro 1. Caracterização da implementação do Nasf nos Municípios do estudo de casos - 2011

\begin{tabular}{|c|c|c|}
\hline & Município A & Município B \\
\hline Localização & Baixada Litorânea & Centro-Sul Fluminense \\
\hline $\begin{array}{l}\text { Faixa populacional } \\
(\mathrm{Hab})\end{array}$ & Menos de 50.000 & De 50.000 a 99.999 \\
\hline $\begin{array}{l}\text { Cobertura saúde da } \\
\text { família }\end{array}$ & $100 \%$ & $94,8 \%$ \\
\hline $\mathrm{N}^{\circ}$ de Nasf & 01 & 02 \\
\hline Início da implementação & 2009 & 2009 \\
\hline Composição Profissional & $\begin{array}{l}\text { - assistente social, educador físico, fisioterapeuta, médico } \\
\text { psiquiatra, médico pediatra, nutricionista, psicólogo, terapeuta } \\
\text { ocupacional. }\end{array}$ & $\begin{array}{l}\text { - assistente social, educador físico, } \\
\text { fisioterapeuta, médico ginecologista, médico } \\
\text { pediatra, nutricionista, psicólogo. }\end{array}$ \\
\hline $\begin{array}{l}\text { Estrutura física e } \\
\text { equipamentos }\end{array}$ & - adequados e satisfatórios. & - apontados como precários. \\
\hline Funcionamento & $\begin{array}{l}\text { - mais relacionado ao gerenciamento e matriciamento; } \\
\text { - deslocamento diário dos profissionais às Unidades de Saúde } \\
\text { da Família (USF). }\end{array}$ & $\begin{array}{l}\text { - mais relacionado ao atendimento individual } \\
\text { especializado; } \\
\text { - deslocamento diário dos profissionais às } \\
\text { USF. }\end{array}$ \\
\hline Organização & $\begin{array}{l}\text { - conhecimento prévio de demandas das equipes de Saúde da } \\
\text { Família; } \\
\text { - elaboração de cronograma mensal de atividades em } \\
\text { conjunto com as EqSF; } \\
\text { - visitas sistematizadas às USF; } \\
\text { - reunião semanal da equipe Nasf. }\end{array}$ & $\begin{array}{l}\text { - reunião da coordenação Nasf com os } \\
\text { profissionais para elaboração de proposta } \\
\text { de ação para cada área de conhecimento de } \\
\text { forma isolada e sem conhecimento prévio das } \\
\text { demandas das EqSF; } \\
\text { - planejamento das atividades realizadas pelos } \\
\text { profissionais; } \\
\text { - reunião mensal para apresentação de } \\
\text { resultados e metas. }\end{array}$ \\
\hline Modelo de atenção & $\begin{array}{l}\text { - integração entre apoio matricial e assistência individual; } \\
\text { - atividades: consultas clínicas individuais, discussão de } \\
\text { casos clínicos, ações clínicas compartilhadas, atividades de } \\
\text { educação permanente para profissionais da ESF, discussões } \\
\text { sobre o processo de trabalho da ESF e do Nasf, elaboração de } \\
\text { intervenções no território em conjunto com as equipes, visitas } \\
\text { domiciliares conjuntas. } \\
\text { - trabalho em equipe em desenvolvimento. }\end{array}$ & $\begin{array}{l}\text { - dicotomia entre apoio matricial e assistência } \\
\text { individual; } \\
\text { - atividades: atendimentos individuais, sala } \\
\text { de espera, atividades em escolas, visitas } \\
\text { domiciliares nem sempre conjuntas; } \\
\text { - o trabalho em equipe não foi destacado. }\end{array}$ \\
\hline $\begin{array}{l}\text { Fatores que favoreceram } \\
\text { a implementação }\end{array}$ & $\begin{array}{l}\text { - necessidades demandadas pelas EqSF para o Nasf; } \\
\text { - aceitação e adesão dos profissionais da ESF à proposta; } \\
\text { - apoio político do gestor da saúde. }\end{array}$ & $\begin{array}{l}\text { - bons profissionais do Nasf; } \\
\text { - empenho da nova coordenação para } \\
\text { aperfeiçoamento da proposta. }\end{array}$ \\
\hline $\begin{array}{l}\text { Fatores que dificultaram } \\
\text { a implementação }\end{array}$ & - desconhecimento da população sobre o Nasf. & $\begin{array}{l}\text { - precário espaço físico das USF e do Nasf; } \\
\text { - desconhecimento da proposta por parte das } \\
\text { EqSF, da população e da rede de saúde; } \\
\text { - salários precários e inferiores aos da ESF; } \\
\text { - forma de contratação dos profissionais. }\end{array}$ \\
\hline
\end{tabular}

Nota: IBGE (dados populacionais), Sala de Apoio à Gestão Estratégica do Ministério da Saúde (Cobertura estimada da Estratégia Saúde da Família) e pesquisa de campo (análise de documentos municipais e entrevistas).

A implementação do Nasf nos municípios estudados ocorreu em período de mudança de governo, devido às eleições municipais em 2008. Houve processos de reformulação da proposta devido às mudanças de atores locais, de forma diferenciada em cada um dos municípios. 
No município A, o processo de implementação do Nasf envolveu diálogo prévio e esclarecimentos junto às EqSF, o que favoreceu a articulação posterior. Na formulação da proposta e determinação da composição profissional do Nasf, a equipe de gestão da $\mathrm{AB}$ contou com a parceria da equipe de saúde mental, que detinha maior conhecimento da lógica de apoio matricial por formação ou experiência profissional. Esses profissionais se capacitaram previamente sobre o conteúdo e os objetivos do Nasf, anterior ao início das atividades nos territórios.

Já no município B, no momento de implementação da proposta, houve dúvidas sobre o caráter de atuação dos núcleos, fundadas numa visão dicotômica sobre as lógicas de apoio matricial versus atendimento direto dos profissionais à população. No momento da pesquisa, o Nasf passava por processo de reformulação ainda incipiente, devido à mudança recente na coordenação, ajustes na organização e iniciativas de capacitação dos profissionais com vistas ao fortalecimento da perspectiva de apoio matricial. Apesar de não haver participação da equipe de saúde mental tão destacada quanto no outro Município A, a articulação do Nasf com essa área tem sido mais fácil do que com as demais, por sua maior compreensão das atividades do núcleo.

A experiência local de implementação no Município A foi reconhecida como positiva para as EqSF pela expectativa de aumento na sua resolutividade. Também favoreceu o compartilhamento de responsabilidades e aprimoramento de estratégias de apoio às equipes. No Município B, a fragilidade de planejamento para a implementação inicial resultou em limitada articulação entre os profissionais dos Nasf e as EqSF.

Portanto, as características do modelo de atenção do Nasf foram diferentes nos Municípios do estudo de casos, expressando influências dos atores locais, a trajetória prévia da $\mathrm{AB}$ e a configuração mais geral do sistema de saúde em cada um. No município A, observou-se a natureza do Nasf vinculada tanto ao apoio matricial quanto aos atendimentos específicos, desde que coordenados pelas EqSF. Já no município B, verificou-se a implementação do Nasf mais direcionada ao atendimento ambulatorial especializado, nos moldes tradicionais. No entanto, no momento da pesquisa, observou-se que a nova equipe gestora se esforçava para fortalecer a ESF e a inserção do Nasf na estratégia.

Em relação à integração do Nasf com outros serviços da rede, os entrevistados dos dois Municípios destacaram seu caráter ainda incipiente e frágil, sugerindo esse aspecto como crítico para o aprimoramento da atuação das equipes de $\mathrm{AB}$ na coordenação do cuidado.

Em ambos os municípios, a atuação do Nasf em uma perspectiva intersetorial ainda é incipiente, sem sistematização ou contatos formais; porém, as áreas da educação e da assistência social foram identificadas como parceiras potenciais.

Como avanços relacionados à implantação do Nasf, foram citados o aprimoramento dos conhecimentos das equipes; a ampliação do escopo da ABS e maior resolutividade da ESF em face das demandas da população.

Em relação às dificuldades de efetivação das regras nacionais do Nasf no âmbito local, identificaram-se: pouca clareza sobre o conteúdo da proposta, a não participação dos atores locais na formulação da proposta local, limitações no cumprimento de carga horária e na educação permanente com os profissionais.

Como desafios para a consolidação do Nasf, destacaram-se maior adesão da população, contratação de profissionais por vínculo estatutário e com melhores salários, resolubilidade das demandas das EqSF e maior articulação dos núcleos com outros serviços de saúde. 


\section{Considerações finais}

O objetivo da pesquisa foi o de contribuir para a reflexão sobre os significados, possibilidades e limites dos Nasf no que concerne ao aprimoramento da $\mathrm{AB}$ em Saúde no Brasil.

$\mathrm{Na}$ esfera federal, a proposta do Nasf reafirmou a saúde da família como porta de entrada e favoreceu o atendimento a demandas presentes no debate nacional de qualificação da $\mathrm{AB}$ : ampliação dos profissionais atuantes na saúde da família, melhoria do cuidado no sentido da integralidade e da capacidade de resolução dos problemas (CASTRO; MACHADO, 2012).

Os dados mostraram que a expansão do Nasf foi expressiva no País, embora desigual no território nacional. O problema de desigualdade na distribuição dos profissionais de saúde no território nacional é estrutural e amplamente reconhecido (CAMPOS; MACHADO; GIRARDI, 2009; DAL POZ, 2013; SCHEFFER, 2013), com repercussões sobre a organização da $\mathrm{AB}$. $\mathrm{A}$ existência de programas e incentivos federais tem sido importante, embora insuficiente, para atenuar esse quadro.

O ritmo de expansão e o perfil dos Nasf foram influenciados pela abrangência populacional prévia das EqSF nas unidades da federação, tanto pelo ritmo de adesão dos municípios ao Nasf como pelas opções dos gestores locais relativas à composição profissional desses núcleos e até mesmo pelas diferenças na disponibilidade dos profissionais das distintas categorias.

Assinale-se que o Nasf é uma proposta recente na $A B$ e tem potencial de expansão adicional nas localidades em que ainda não se atingiu a quantidade de Nasf recomendada pela regulamentação federal para apoiar as EqSF existentes ou para ser acoplada à implementação de novas EqSF.

$O$ perfil de composição profissional dos Nasf em nível nacional, marcado pelo predomínio de profissionais não médicos, reforça a importância da influência da reivindicação de outras categorias profissionais de saúde para a inserção na ESF. Nesse sentido, o Nasf possibilitou uma ampliação do mercado de trabalho na $\mathrm{AB}$ para várias categorias profissionais atuantes na área da saúde. A inserção dos médicos foi maior nas especialidades tradicionalmente atuantes na $\mathrm{AB}$ - como pediatras, ginecologistas e obstetras - e pouco expressiva no que se refere aos psiquiatras e às especialidades de homeopatia e acupuntura, talvez por seu menor número no País e pelo caráter mais recente da Política Nacional de Práticas Integrativas e Complementares no SUS.

No que se refere à implantação do Nasf no estado do Rio de Janeiro, sua condição está relacionada à organização da $\mathrm{AB}$ em saúde no Estado, que, por sua vez, apresenta as características da descentralização da política de ABS no País, as especificidades do Estado e as dificuldades apresentadas pelo gestor estadual na coordenação do sistema. Assim, a implementação do Nasf nos municípios tem sido diversificada, de acordo com as distintas condições de gestão dos sistemas locais e as decisões políticas dos atores municipais. De qualquer forma, no estado do Rio de Janeiro - diferentemente dos dados referentes ao conjunto do País, regiões e UF - a maioria dos municípios está na faixa de 8 a 15 EqSF para cada Nasf recomendada pelo Ministério da Saúde.

No Estado, o envolvimento da equipe de saúde mental da SES/RJ na análise dos projetos municipais e implementação do Nasf, em articulação com a equipe da Superintendência de Atenção Básica, pode ter influenciado a presença mais frequente do profissional psicólogo na composição dos Nasf. A influência da equipe técnica de saúde mental, registrada na formulação nacional da proposta e na esfera estadual, também se mostrou com maior destaque em um dos municípios pesquisados.

Quanto à implementação local do Nasf, estudos de caso realizados em outras localidades do País podem contribuir para a reflexão neste trabalho. Uma primeira questão refere-se às lacunas na regulamentação da proposta pelo Ministério da Saúde. Sampaio et al. (2012) abordam em estudo de 
implantação do Nasf em Campina Grande/ PB que, no momento inicial, os documentos federais deixaram margem a uma diversidade de interpretações sobre seu papel, uma vez que o conceito de apoio matricial foi indicado apenas em 2009 (BRASIL, 2009), um ano após o lançamento da proposta. Assim, cada Nasf local compôs seu trabalho de acordo com sua própria compreensão de matriciamento. Nascimento (2014) afirma que essa dificuldade de direcionamento inicial da proposta permitiu que os Nasf do Recife (PE) se tornassem flexíveis para atender às demandas locais devido aos diversos modelos de atenção em disputa.

Nesse sentido, uma segunda questão inter-relacionada à primeira diz respeito à variedade de características que os Nasf apresentam a depender da região em que estão inseridos, das equipes que os compõem e do perfil das EqSF. Segundo Sampaio et al. (2012) as disputas no contexto local sobre como operacionalizar o Nasf em Campina Grande (PB) permitiram a conformação de diferentes modelos segundo as escolhas das gestões locais de saúde.

Em Recife (PE), a variabilidade de contextos locais permitiu que cada gestão municipal direcionasse a forma de organização da atenção à saúde pelo Nasf e seu processo de trabalho, configurando-os por uma influência de fatores. A indefinição quanto ao objeto, atenção especializada ou apoio matricial, bem como precariedades nas condições de trabalho, teria dificultado a organização do processo de trabalho na implementação dos Nasf nessa localidade (NASCIMENTO, 2014).

No entanto, a tensão entre apoio matricial e atendimento individual, também registrada na pesquisa de campo do Rio de Janeiro, pode ser considerada um falso dilema, uma vez que, na proposta do Nasf, a assistência individual não é incompatível com o apoio matricial. Os profissionais dos núcleos podem realizar os dois tipos de ações, desde que a coordenação do cuidado seja das EqSF. A ênfase no apoio matricial, no entanto, é marcante na proposta nacional do Nasf, talvez pela preocupação de seus formuladores em afirmar os núcleos como integrantes da ESF com vistas à mudança do modelo de atenção e não somente à ampliação da oferta de atendimentos especializados.

Para Lacman et al. (2013), em estudo de caso sobre o trabalho do Nasf em São Paulo, as ferramentas tecnológicas utilizadas pela proposta, tais como o apoio matricial e o projeto terapêutico singular, são recentes na $\mathrm{AB}$ e precisam ser consolidadas. Porém, é necessário que os profissionais inseridos tenham disponibilidade para realizar um trabalho mais compartilhado.

Nos estudos de caso dos municípios fluminenses, verificou-se que a implementação no Rio de Janeiro caminha pari passu aos estudos apresentados em outras localidades do País, com modelos de atenção configurados conforme as influências de atores locais, trajetória prévia da $\mathrm{AB}$ e do sistema de saúde de uma forma geral. Porém, cabe destacar que o papel de parceria da equipe da saúde mental no processo de implementação do Município A facilitou a lógica do apoio matricial na intervenção do Nasf desde o início de sua implementação. Já no Município B, em que tal parceria não aconteceu de forma tão próxima, o momento inicial do Nasf local caracterizou-se pela dicotomia entre apoio matricial e atenção especializada, com limitada articulação dos dois tipos de práticas.

A integração com a rede de saúde foi apresentada como incipiente e frágil nos municípios fluminenses pesquisados, o que indica um longo caminho a ser percorrido no que concerne a um dos principais propósitos do Nasf: favorecer a $\mathrm{AB}$ resolutiva, coordenadora do cuidado e articulada com os outros níveis de atenção.

Quanto aos limites da pesquisa, vale assinalar o caráter ainda recente da implantação dos núcleos, a realização do trabalho de campo em momento de troca de dirigentes, as dificuldades de obtenção de dados sistematizados sobre a atuação dos Nasf nos 
municípios e a realização de um número de entrevistas relativamente restrito. Os estudos de caso tiveram caráter exploratório e levantaram questões que requerem investigações específicas, tais como: o processo de trabalho desses núcleos, suas relações com as EqSF, as implicações da incorporação de outros profissionais para a atenção em áreas específicas do cuidado, os resultados da atuação dos Nasf em termos da ampliação do acesso, da integralidade e da qualidade da atenção, entre outras.

Em termos gerais, o estudo revelou que o processo de implementação do Nasf desvenda questões ainda mal equacionadas

\section{Referências}

BRASIL. Ministério da Saúde. Política Nacional de Atenção Básica. Brasília, DF: Ministério da Saúde, 2006. (Série Pactos pela Saúde 2006, v. 4).

Ministério da Saúde. Portaria $M S / G M n^{\circ} 154$, de 24 de janeiro de 2008. Cria os Núcleos de Apoio à Saúde da Família. Diário Oficial [da] União. Brasília, DF, 2008. Disponível em: <https://www.google.com.brrl?sa=t\&r $\mathrm{ct}=\mathrm{j} \& \mathrm{q}=\&$ esrc $=\mathrm{s} \&$ source $=$ web $\& \mathrm{~cd}=1 \& \mathrm{ved}=0$ ahUKEwjy uPne5brJAhWFn5AKHdKUBBQQFggcMAA\&url=htt p\%3A\%2F\%2Fdab.saude.gov.br\%2Fdocs\%2Flegislaca o\%2Fportarial54_24_01_08.pdf\&usg=AFQjCNFG3RN SVyBLNAyrkk0j194rkak6zQ\&sig2=yilgMj5hzkxxag9 gO_qUaQ>. Acesso em: 15 out. 2015.

Ministério da Saúde. Portaria MS/GM nº 2.843, de 20 de setembro de 2010. Cria, no âmbito do Sistema Único de Saúde - SUS, os Núcleos de Apoio à Saúde da Família - Modalidade 3 - NASF 3, com prioridade para a atenção integral para usuários de crack, álcool e outras drogas. Diário Oficial [da] União. Brasília, DF, 2010.

Disponível em: <http://bvsms.saude.gov.br/bvs/saudelegis/gm/2010/prt2843_20_09_2010.html>. Acesso em: 15 out. 2015. no âmbito da $\mathrm{AB}$ e fragilidades da gestão pública em saúde que extrapolam esse nível de atenção e são concernentes às relações entre esferas de governo, às condições de inserção e de trabalho dos profissionais, à integração com o restante da rede de serviços e com outras áreas da política pública, entre outras. O enfrentamento dessas questões é fundamental para o fortalecimento efetivo da $\mathrm{AB}$ como eixo estruturante do sistema de saúde brasileiro, conforme formalmente anunciado nas diretrizes nacionais do SUS, mas nem sempre garantido nas condições reais de implementação das políticas. Saúde. Departamento de Atenção Básica. Diretrizes no NASF: Núcleo de Apoio a Saúde da Família. Brasília, DF: Ministério da Saúde, 2009. (Cadernos de Atenção Básica, n² 27).

Ministério da Saúde. Secretaria de Atenção à Saúde. Departamento de Atenção Básica. Política Nacional de Atenção Básica. Brasília, DF: Ministério da Saúde, 2012. (Série E. Legislação em Saúde).

CAMPOS, G. W. S; DOMITTI, A. C. Apoio Matricial e Equipe de Referência: uma metodologia para a gestão do trabalho interdisciplinar em saúde. Cad. Saúde Pública, Rio de Janeiro, v. 23, n. 2, p. 399-407, fev. 2007.

CAMPOS, F. E.; MACHADO, M. H.; GIRARDI, S. N. A fixação de profissionais de saúde em regiões de necessidades. Divulgação em Saúde para Debate, Rio de Janeiro, n. 44, p. 13-24, 2009.

CASTRO, A. L. B.; MACHADO, C. V. A política federal de atenção básica à saúde no Brasil nos anos 2000.

Physis, Rio de Janeiro, v. 22, n. 2, p. 477-506, 2012. 
DAL POZ, M. R. A crise da força de trabalho em saúde. Cadernos de Saúde Pública, Rio de Janeiro, v. 29, n. 10, p. 1924-1926, 2013.

GÉRVAS, J. Atención Primaria de Saluden Europa: tendencias a princípios Del siglo XXI. Una reflexión con motivo de los XXV años de La Declaración de Alma Ata. Artículo de Opinión. SEMERGEN, Madrid v. 30, n. 5, p. 245-57, 2004.

LACMAN, S. et al. Estudo do Trabalho e do Trabalhar no Núcleo de Apoio à Saúde da Família. Revista Saúde Pública, São Paulo, v. 47, n. 5, p. 968-75, 2013.

NASCIMENTO, C. M. B. Núcleo de Apoio à Saúde da Família: uma análise da atenção à saúde em municípios da Região Metropolitana do Recife. 2012. Tese (Doutorado em Saúde Pública) - Fundação Oswaldo Cruz, Recife, 2014.

SAMPAIO, J. et al. O NASF como dispositivo da gestão: limites e possibilidades. Revista Brasileira de Ciências da Saúde, João Pessoa, v.16, n. 3, p. 317-324, 2012.

SCHEFFER, M. (Org.). Demografia Médica no Brasil. São Paulo: Conselho Regional de Medicina do Estado de São Paulo: Conselho Federal de Medicina, 2013. v. 2.
STARFIELD, B. Atenção Primária: equilíbrio entre necessidades de saúde, serviços e tecnologia. Brasília, DF: UNESCO, Ministério da Saúde, 2004.

\section{VIANNA, R. P.; LIMA, L. D. Colegiados de Gestão} Regional no estado do Rio de Janeiro: atores, estratégias e negociação intergovernamental. Physis, Rio de Janeiro, v. 23, n. 4, p. 1025-1049, 2013.

VUORI, H. Health for all, primary health care and general Practitioners. Journal of the Royal College of General Practitioners, London, n. 36, p. 398-402, sept. 1986. Disponível em: <http://www.pubmedcentral. nih.gov/picrender.fcgi? artid=1960528\&blobtype $=$ pdf $>$. Acesso em: 10 ago. 2010.

WALT, G. Health Policy: an introduction to process and power. London: Zed Books, 1994.

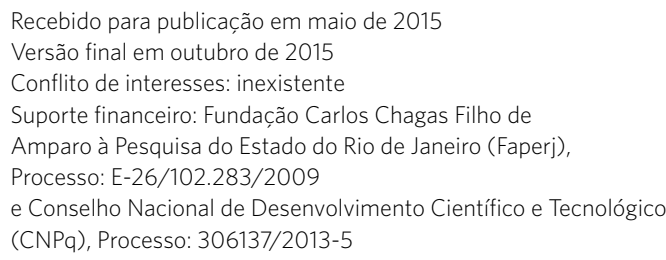

